\title{
Emergency Management of Refractory Hypoxemia in Mechanically Ventilated Patients with COVID-19 Acute Respiratory Distress Syndrome
}

\author{
Subash P. Nandalan, Mathew Patteril ${ }^{1}$ \\ Department of Anaesthesia and Intensive Care Medicine, Royal Albert Edward Infirmary, Wigan, UK, 'Department of Perioperative Medicine, \\ Pain Management and Critical Care University Hospitals Coventry and Warwickshire NHS Trust, Coventry, UK
}

\section{Abstract}

Covid-19 disease has had a significant impact on intensive care facilities worldwide. In ventilated patients with Covid-19 ARDS, refractory hypoxemia is a particularly challenging scenario. This article presents a case study and explores a systematic approach to the management of refractory hypoxemia. Current evidence base for Covid-19 medical management is discussed. It is emphasized that one must adhere to evidence based principles of ARDS management.

Keywords: COVID-19, refractory hypoxemia, ventilation

\section{INTRODUCTION}

The novel severe acute respiratory syndrome coronavirus 2 infection, clinically manifested as coronavirus disease 2019 (COVID-19), has had a significant impact on intensive care facilities worldwide. The need for ventilatory support for acute respiratory distress syndrome (ARDS) has been the predominant feature in those who required intensive care unit (ICU) admission. While a significant proportion of these patients improved with noninvasive measures, the mechanically ventilated patients had a poorer outcome with mortality rates quoted between $20 \%$ and $40 \%$. Refractory hypoxemia in such mechanically ventilated patients is a complex and difficult-to-manage medical emergency.

\section{Definition OF HyPOXEMIA}

As per the ARDS Net mechanical ventilation protocol, the oxygenation goal is accepted as $\mathrm{PaO}_{2} 7.3-10.5 \mathrm{kPa}$ (55-80 $\mathrm{mmHg}$ ), or $\mathrm{SpO}_{2}$ of $88 \%-95 \%$. In our practice, we accept a $\mathrm{PaO}_{2}$ of $8 \mathrm{kPa}(60 \mathrm{mmHg})$ as acceptable oxygenation in a mechanically ventilated ARDS patient.

\begin{tabular}{|l|l|}
\hline \multicolumn{3}{|c|}{ Access this article online } \\
\hline Quick Response Code: & Website: \\
\hline & www.ijrc.in \\
\hline
\end{tabular}

\section{"Happy Hypoxemia" (SILent HypoXemia) in COVID-19}

The remarkable dissociation between severe arterial hypoxemia and the relatively comfortable appearance of the patient has been described as "happy hypoxemia." It is important for clinicians to not be fooled by the apparent wellness, but to closely monitor for signs of fatigue by regular monitoring of respiratory rate, oxygen saturation, and arterial blood gases. Arterial hypoxemia is caused by intrapulmonary shunting, dysregulated hypoxic pulmonary vasoconstriction, impaired lung diffusion, and intravascular microthrombi formation. In the initial stages, there is no increased airway resistance or dead space ventilation because the lung mechanics are well preserved. As a result, the respiratory center does not sense an uncomfortable sensation of breathing, resulting in a "happy" appearance. However, sudden and rapid respiratory

Address for correspondence: Dr. Subash P. Nandalan, Consultant in Anaesthesia and Intensive Care Medicine Royal Albert Edward Infirmary, Wigan WN1 2NN, UK. E-mail: spnandalan@gmail.com

This is an open access journal, and articles are distributed under the terms of the Creative Commons Attribution-NonCommercial-ShareAlike 4.0 License, which allows others to remix, tweak, and build upon the work non-commercially, as long as appropriate credit is given and the new creations are licensed under the identical terms.

For reprints contact: WKHLRPMedknow_reprints@wolterskluwer.com

How to cite this article: Nandalan SP, Patteril M. Emergency management of refractory hypoxemia in mechanically ventilated patients with COVID-19 acute respiratory distress syndrome. Indian J Respir Care 2021;10:S64-9.

Received: $21-01-2021$ Revised: 04-03-2021

Accepted: 10-03-2021 Published: 29-04-2021 
decompensation may occur and the patient should be closely monitored.

\section{Progression of Disease and Respiratory Support for the Hypoxic Patient}

COVID-19 ARDS (CARDS) was described by Gattinoni et al., ${ }^{[1]}$ using a phenotypical approach as type $\mathrm{L}$ and type $\mathrm{H}$. The L-type was described with normal to high pulmonary compliance, low lung elastance, low lung weight, and low lung recruitability. The H-type was described as typical ARDS with severe hypoxemia, markedly reduced pulmonary compliance, high lung elastance, high lung weight, and high lung recruitability. They did not identify these as two separate pathological entities, but simply as earlier and later stages of CARDS disease progression. The pathophysiology of CARDS is not dissimilar from classical ARDS. Different phenotypes with variable lung compliance depending on the disease stage have been proposed and the phenotypical difference is also seen with classical ARDS. It is also important to observe that the clinical manifestation of CARDS conforms to the Berlin definition ${ }^{[2]}$ of ARDS, regardless of the phenotypical stage of the disease process. The only evidence-based ventilation approach with mortality benefit is the low tidal volume protective lung ventilation $^{[3]}$ and it is also beneficial to use this approach even where the lung condition does not conform to ARDS definitions.

Noninvasive modalities of respiratory support are efficacious and may reduce the requirement for invasive ventilation. In contrast to earlier recommendations, noninvasive ventilation (NIV) and high flow nasal cannula are often effectively employed as the initial modality of respiratory support. Decisions regarding the timing of endotracheal intubation and invasive mechanical ventilation must be individualized. It is important to diligently monitor patients who are on noninvasive support and identify those who show signs of deterioration early. At our institution, we consider the following as "red flags" to prompt consideration for endotracheal intubation: $\mathrm{PaO}_{2} / \mathrm{FiO}_{2}$ ratio $<12 \mathrm{kPa}(90 \mathrm{mmHg})$ and respiratory rate $>30$ breaths $/ \mathrm{min}$ for more than $2 \mathrm{~h}$ despite adequate anxiolysis in a context of deteriorating clinical trajectory. Once mechanical ventilation is commenced, the time-tested protective lung ventilation strategy ${ }^{[3]}$ of low tidal volumes and titrated PEEP with limitation of driving pressures would be appropriate. The timing of tracheostomy also needs individualization. Tracheostomy should be considered in patients who may require long-term ventilation and carry a high risk of extubation failure. $\mathrm{The}^{\mathrm{FiO}}{ }_{2}$ requirement should be $\leq 0.4$ with a PEEP requirement of $\leq 10 \mathrm{cmH}_{2} \mathrm{O}$ before tracheostomy is contemplated.

\section{Refractory Hypoxemia in the Mechanically Ventilated COVID-19 Patient}

The management of hypoxemia in CARDS must follow the general principles of managing any ventilated ARDS patient, but always supported and reinforced by the knowledge of specific pathophysiology and related complications in COVID-19.

Refractory hypoxemia occurs in four distinct scenarios in CARDS.

1. During the initial establishment of mechanical ventilation

2. Related to COVID-19 disease progression-cytokine storm episodes

3. Related to ventilator asynchrony

4. As a result of complications related to mechanical ventilation

5. As a result of other pulmonary complications.

\section{Case Study}

A 52-year-old previously fit and well man who had been an inpatient for 5 days with a clinical diagnosis of COVID-19 has been receiving NIV for the previous $24 \mathrm{~h}$. Despite some initial improvement, his $\mathrm{PaO}_{2} / \mathrm{FiO}_{2}$ ratio dropped to $13 \mathrm{kPa}(100$ $\mathrm{mmHg}$ ), and he became increasingly tachypneic and showed signs of fatigue. His respiratory features conformed to the Berlin definition of ARDS. The decision was made to perform endotracheal intubation and start mechanical ventilation. The oxygen saturation by pulse oximetry at this point was measuring $85 \%$, and it did not improve above $92 \%$ despite preoxygenation with a Waters circuit delivering 100\% oxygen. During the process of endotracheal intubation, he became extremely hypoxic with oxygen saturation dropping to $50 \%$. The oxygen saturation slowly improved to $90 \%$ after ventilating manually with a Waters circuit through the endotracheal tube. Mechanical ventilation was initiated with Bilevel-Volume Guaranteed (Bilevel-VG) mode using the principles of low tidal volume protective lung ventilation strategy. With an initial high PEEP setting of $15 \mathrm{cmH}_{2} \mathrm{O}$, his $\mathrm{PaO}_{2}$ gradually improved over the next $24 \mathrm{~h}$, achieving a $\mathrm{FiO}_{2}$ setting of 0.4 with $\mathrm{a} \mathrm{PaO}_{2} / \mathrm{FiO}_{2}$ ratio of $20 \mathrm{kPa}$.

Despite the initial rapid improvement, the oxygenation deteriorated on multiple occasions during his ICU stay totaling 38 days. Initially, the hypoxic episodes resolved by adjusting ventilator settings, airway clearance, muscle relaxant infusion, and prone positioning. He stopped responding to prone positioning after four episodes over 2 weeks. In the initial period, the airway heat and moisture exchanger (HME) filter was replaced on multiple occasions due to clogging. In the latter half of his ICU stay, he suffered an episode of tension pneumothorax requiring emergency chest drain insertion. A percutaneous tracheostomy was inserted on day 21 and he was gradually weaned off the ventilator. Ventilator weaning was significantly prolonged due to hyperactive delirium and critical illness polyneuropathy and myopathy.

\section{A Systematic Approach to Managing Hypoxemia in the Ventilated CARDS Patient}

A systematic check must always be performed and causes narrowed down when dealing with refractory hypoxemia in all the above scenarios. 


\section{Establishing an Open Airway and Oxygen DeLIVERY}

In all mechanically ventilated patients, a thorough check is performed to establish that the ventilator circuit, HME filter and the endotracheal tube (or tracheostomy tube) are patent and in continuity. Clinical examination of the patient, physical examination of these airway components and evaluation of the end-tidal $\mathrm{CO}_{2}$ information should be conducted as a priority. Endobronchial migration of the tracheal tube is an easily corrected, but common scenario, especially during nursing maneuvers. The presence of excessive respiratory secretions is a common feature in CARDS and this could result in clogging of the endotracheal tube (or tracheostomy) and the HME filter. In our institution, we had to replace these devices to establish a clear airway on multiple occasions. It was common to find significant improvement in airway pressures after these device exchanges.

Always check that the ventilator is delivering the set oxygen percentage by checking the monitored inspired gas oxygen measurement. As an immediate measure, inspired oxygen concentration may be increased appropriately to counter hypoxemia.

\section{Checking Ventilator Settings and Assessing Ventilator Asynchrony}

We have found that sudden alteration in airway mechanics resulted in mechanical ventilators resetting to their backup mode. This was a common, but detected late, cause of hypoxemia in CARDS patients due to the abrupt changes in ventilatory mechanics they suffered.

Finding the appropriate and effective ventilator settings is challenging especially during the initial stages. Regardless of the choice of ventilation mode, it must conform to evidence-based ARDS management. Low tidal volume protective lung ventilation ${ }^{[3]}$ is the only approach that is proven to have a mortality benefit in a large randomized controlled trial. There have been multiple clinical trials that showed it is a safer approach even in patients without ARDS. The patient's predicted weight, based on sex and height, should be calculated and a tidal volume between 4 and $8 \mathrm{ml} / \mathrm{kg}$ achieved, but not exceeded. Given the variable lung mechanics in CARDS (described as L type and $\mathrm{H}$ type by Gattinoni et $\left.a l^{[1]}\right)$, the PEEP may be appropriately set at low levels in the initial stages. A higher PEEP approach may be necessary when lung mechanics conform to the $\mathrm{H}$ phenotype. We found the Bilevel-VG mode useful in keeping to the recommended tidal volumes. Where possible we must ensure that the plateau airway pressure does not exceed $30 \mathrm{cmH}_{2} \mathrm{O}$.

Some clinicians have used the airway pressure release ventilation (APRV) mode of ventilation to manage hypoxemia. This mode maintains a high airway pressure and achieves tidal volume by releasing this pressure periodically. Patients can breathe spontaneously during any phase of the APRV ventilation without the need for deep sedation. We use this mode as rescue mode where Bilevel mode and prone positioning fails to improve hypoxemia, or where patients fail to tolerate conventional modes of ventilation. It is also useful in patients with contraindications to prone positioning. It is important to note however that there is no reliable way of controlling the tidal volumes or airway pressures accurately for spontaneous breaths taken during APRV. Therefore, reckless use of APRV can be counterproductive and worsen the CARDS or cause barotrauma.

Patients in the prone position need to be adequately sedated while they are paralyzed. We have found that despite a large dose of sedative infusion and muscle relaxant infusion, ventilator asynchrony has occurred in our patients. Increasing the rate of these infusions or even adding a new sedative may resolve ventilator synchrony.

\section{Assess the State of the Tracheobronchial Tree}

Bronchoscopy to clear the tracheobronchial tree can prove very successful in resolving hypoxemic episodes. We found that many CARDS patients produced excessive respiratory mucus secretions which formed mucus plugs that blocked the tracheobronchial tree. These were too thick to be dislodged by closed endotracheal suctioning. Bronchoscopy has the additional benefit of being able to collect microbiological and serological samples with higher diagnostic yield.

\section{Recruitment Maneuvers}

The COVID-19 disease affects the lungs with direct cytopathic damage of the pneumocytes and virus-induced decrease in surfactant levels causing atelectasis. In addition, diffuse alveolar hemorrhage and hyaline membrane formation have also been described. Although the early (L-type) COVID-19 lung pathology is not amenable to recruitment, the later (H-type) lung pathology does respond to recruitment maneuvers. It is probable that patients who failed noninvasive respiratory support and progressed mechanical ventilation belonged to the second category. Therefore, it is justified to perform recruitment maneuvers and manage it as per traditional ARDS management with high PEEP to maintain recruitment of alveoli. However, given the widespread and extensive lung damage in COVID-19, the clinician should be extra cautious to avoid barotrauma resulting from overzealous recruitment maneuvers.

\section{Radiological Evaluation - Chest X-ray, Lung Ultrasound, Bedside Echocardiogram, Computed TOMOGRAPHY THORAX}

Critically ill CARDS patients have an increased incidence of respiratory complications related to mechanical ventilation and invasive procedures. The predominant complication has been pneumothorax due to ventilator-induced barotrauma or as a complication of central venous line insertion. We have 
also come across CARDS patients presenting with computed tomography (CT) thorax evidence of extensive emphysematous bullae formation, in the absence of previous respiratory disease. Such bullae could rupture when exposed to high airway pressures, especially during recruitment maneuvers. Hypoxemia in association with circulatory collapse should alert the clinician to the possibility of a tension pneumothorax.

We have also come across multiple cases of pneumomediastinum in patients receiving invasive as well as NIV. Some of these were isolated while others had associated pneumothorax requiring chest drains or surgical emphysema. Isolated pneumomediastinum or surgical emphysema is of great clinical concern, but not easily amenable to drainage. The presence of pneumomediastinum suggests barotrauma to a mediastinal structure such as the major airways. Extreme caution is necessary when positive pressure ventilation is managed in such cases.

We have also come across an incidence of significant hemothorax formation in a ventilated obese patient who was also receiving therapeutic anticoagulation for pulmonary embolic disease with COVID-19. In this case, there was hypoxemia in association with sudden unexplained anemia and circulatory collapse.

A chest X-ray must be performed for additional diagnostic information, especially if the hypoxemia does not resolve with the above measures. Emergency chest drain insertion can resolve the situation, but not without complications. We have come across two cases of persistent bronchopleural fistula during the first surge of COVID-19 at our institution.

Bedside lung ultrasound is an extremely useful diagnostic tool that can quickly diagnose pneumothorax and pleural effusions. It is also useful in assessing the progression of COVID-19-related lung changes. Bedside echocardiography can diagnose and differentiate between the causes of circulatory collapse-hypovolemia, left/right ventricular impairment, or pulmonary embolism. Both these assessments using a bedside ultrasound machine can be performed while waiting for the radiographer to arrive with the portable X-ray machine. It is a safe and quick modality to yield vital information for optimizing the patient's condition.

We routinely perform a CT-pulmonary angiogram (CT-PA) before admitting the COVID-19 patient to the ICU for mechanical ventilation. A follow-up CT-PA after the resolution of refractory hypoxemia will provide valuable information. Pulmonary embolism is very common in COVID-19 and its presence mandates therapeutic anticoagulation as per our local protocol. In addition, CT-PA would provide information on the extent and progression of COVID-19 lung damage and its pattern. The latter information is extremely helpful in the management of refractory hypoxemia, and consideration for prone positioning.

\section{Prone Positioning}

CARDS hypoxia responds well to prone positioning. This intervention is beneficial in the awake patient receiving noninvasive respiratory support, as well as in the sedated mechanically ventilated patients. Response to prone positioning occurs even in the later stages of the disease. Patients with a predominantly posterior distribution of COVID-19 pulmonary damage are the most likely to benefit from prolonged prone positioning. This will improve ventilation to the posterior regions and improve both ventilation-perfusion mismatch and reduce pulmonary shunting effect. We use the local protocol for proning the patients with the help of a designated "proning team," a team of six assistants led by a clinician in control of the airway. Each episode of proning is aimed to be maintained for 16 $\mathrm{h}$ at a time (maximum $24 \mathrm{~h}$ ). There is a variable length of time before patients respond to prone positioning, hence the prolonged duration. We have observed that after several such episodes some patients stop responding meaningfully to proning any further. This might mean that the progression of their COVID-19 pulmonary damage pattern is no longer responsive to the benefits of prone positioning.

\section{Circulatory and Cardiac Causes of Refractory HYPOXEMIA}

Intrapulmonary shunting as well pulmonary microthrombi formation has been described in COVID-19 pathophysiology. The former relates to continued pulmonary circulation into areas with collapsed alveoli, whereas the latter pathology causes a dead space effect whereby normal alveoli are rendered unable to take part in gas exchange by the absence of circulation. Intrapulmonary shunting may be reversed to some extent by recruitment maneuvers and high PEEP as part of the mechanical ventilation strategy. The pulmonary microthrombi formation is much harder to reverse and it could be part of the larger pulmonary embolization effect. This would form the rationale for treating patients with therapeutic anticoagulation. The current guidelines advise therapeutic anticoagulation only where there is evidence of pulmonary or systemic embolic phenomena. In other situations, only prophylactic anticoagulation is recommended.

Bedside and formal echocardiograms have been very useful in COVID-19 management. We have uncovered a range of cardiac functional abnormalities ranging from hypovolemia, left ventricular dysfunction, right ventricular dysfunction, global dysfunction, and pericardial effusion. A low cardiac output state could result from COVID-19 viral myocarditis or due to the myocardial dysfunction from sepsis due to any secondary infections. Increased cardiac output and increased oxygen demand due to sepsis can also be present. All of these would contribute to hypoxemia unless managed appropriately.

\section{Restrictive Fluid Strategy and Appropriate Use OF Muscle Relaxation}

There is evidence to show that a restrictive fluid strategy is beneficial in patients with ARDS provided there is no 
circulatory or renal impairment. Where appropriate, we use a furosemide infusion to target urine output of $100 \mathrm{ml} / \mathrm{h}$.

Similarly, appropriate use of muscle relaxant infusion can facilitate mechanical ventilation and improve outcomes in ARDS. This benefit must be balanced against the risks from prolonged muscle relaxant use, mainly that of critical illness polyneuropathy and myopathy. After the initial 48 $h$, its use should be limited to emergency management of hypoxemia. Where appropriate we use a muscle relaxant infusion to facilitate ventilator synchrony and to aid with prone positioning. It is argued that the use of APRV ventilation mode can significantly reduce the use of muscle relaxants.

\section{Pulmonary Vasodilator Therapy, Extracorporeal Membrane Oxygenation}

Inhaled nitric oxide therapy and inhaled prostacyclin have been described as rescue therapies in ARDS, but with no mortality benefit. These modalities are not commonly available and would only serve as bridging measures for patients waiting for extracorporeal membrane oxygenation (ECMO). We refer patients who remain in refractory hypoxemia to the local ECMO center who runs a retrieval service for patients who meet their selection criteria. ECMO improves hypoxemia through extracorporeal oxygenation, while the underlying COVID-19-related lung damage recovers. Using ECMO in COVID-19 is a challenge and evidence is still lacking to recommend its routine use.

\section{Appropriate Drug Therapy in COVID-19}

Based on the available evidence we treat all our COVID-19 patients with low dose dexamethasone (6 mg once daily for 10 days). We also administer interleukin 6 receptor antagonists tocilizumab or sarilumab in the absence of contraindications and within $24 \mathrm{~h}$ of starting organ support. Evidence ${ }^{[4,5]}$ suggests that both tocilizumab and sarilumab are beneficial in adults with severe COVID-19 who are critically ill and receiving respiratory or cardiovascular organ support in an intensive care setting. Either of these drugs must be given within about $24 \mathrm{~h}$ of starting organ support. Usage outside this period is not currently recommended given the increased risk of side effects and secondary infections in COVID-19.

In critically ill patients, the current guidelines advise therapeutic anticoagulation only where there is evidence of pulmonary or systemic embolic phenomena. In other situations, only prophylactic anticoagulation is recommended. The UK National Institute for Health and Care Excellence (NICE) ${ }^{[6]}$ advise that consideration should be given to increasing the standard prophylactic dose of parenteral anticoagulation, such as LMWH, to an intermediate dose to mitigate the increased risk of VTE while minimizing the risk of bleeding associated with higher doses. In line with the NICE guideline, our critically ill patients routinely receive intermediate dosing in the absence of contraindications. The current evidence is in favor of therapeutic anticoagulation for moderately ill COVID-19 patients (before they become critically ill). Long-term anticoagulation is still a gray area and current guidelines only recommend using oral anticoagulation after hospital discharge for those who had a proven thromboembolic event.

In the absence of survival benefit, we no longer routinely administer remdesivir to our critically ill patients. The ACTT-1 trial $^{[7]}$ suggested that remdesivir administration may reduce the hospital length of stay if administered within the first 10 days of onset of symptoms among less severely ill patients. No mortality benefit was demonstrated with remdesivir in the ACTT-1 trial. A trend toward improved survival by day 29 was observed in patients who were only on supplemental oxygen, without the requirement for mechanical ventilation. The Solidarity trial ${ }^{[8]}$ also did not reveal improved survival among patients who received remdesivir; furthermore, in contrast to the ACTT-1 trial, the number of patients who continued to receive hospitalized care did not differ with remdesivir compared to the control group at days 7, 14, and 21. Detailed information regarding the severity of illness at baseline, the time point of remdesivir administration during the illness, and information regarding other treatment modalities employed were not provided in the Solidarity trial. It is plausible that early administration of remdesivir to moderately ill patients may improve recovery. However, future controlled trials among subgroups of patients with less severe illness are required to confirm this hypothesis.

The Solidarity trial ${ }^{[8]}$ also showed that along with remdesivir, hydroxychloroquine, lopinavir, and interferon regimens had little or no effect on hospitalized patients with COVID-19, as indicated by overall mortality, initiation of ventilation, and duration of hospital stay. We also no longer administer convalescent plasma on the basis that there has been no convincing evidence of the effect of convalescent plasma on clinical outcomes in patients admitted to hospital with COVID-19. ${ }^{[9]}$ For patients with severe CARDS, we use methylprednisolone regime(s) at clinical discretion to improve pulmonary recovery, but the evidence is not conclusive in this regard.

In addition, we treat any proven or suspected secondary infection with appropriate antibiotics, guided by infection markers (including procalcitonin), and consultant microbiologist advice.

\section{ConcLusion}

A systematic approach to managing refractory hypoxemia in the CARDS is described. It is emphasized that one must adhere to principles of evidence-based ARDS management while managing CARDS patients.

\section{Financial support and sponsorship}

Nil.

Conflicts of interest

There are no conflicts of interest. 


\section{REFERENCES}

1. Gattinoni L, Chiumello D, Caironi P, Busana M, Romitti F, Brazzi L, et al. COVID-19 pneumonia: Different respiratory treatments for different phenotypes? Intensive Care Med 2020;46:1099-102.

2. ARDS Definition Task Force; Ranieri VM, Rubenfeld GD, Thompson BT, Ferguson ND, Caldwell E, et al. Acute respiratory distress syndrome: The berlin definition. JAMA 2012;307:2526-33.

3. Acute Respiratory Distress Syndrome Network; Brower RG, Matthay MA, Morris A, Schoenfeld D, Thompson BT, et al. Ventilation with lower tidal volumes as compared with traditional tidal volumes for acute lung injury and the acute respiratory distress syndrome. N Engl J Med 2000;342:1301-8.

4. National Institute for Health and Care Excellence. COVID-19 Rapid Evidence Summary: Tocilizumab for COVID-19; 2021. Available from: https://www.nice.org.uk/advice/es33/chapter/Product-overview. [Last accessed on 2021 Mar 01].

5. National Institute for Health and Care Excellence. COVID-19 Rapid Evidence Summary: Sarilumab for COVID-19; 2021. Available from:
https://www.nice.org.uk/advice/es34/chapter/Product-overview. [Last accessed on 2021 Mar 01].

6. National Institute for Health and Care Excellence. COVID-19 Rapid Guideline: Reducing the Risk of Venous Thromboembolism in Over 60s with COVID-19 (NG186); 2021. Available from: https://www.nice.org. uk/guidance/ng186. [Last accessed on 2021 Mar 01].

7. Beigel JH, Tomashek KM, Dodd LE, Mehta AK, Zingman BS, Kalil AC, et al. Remdesivir for the treatment of COVID-19 - Final report. N Engl J Med 2020;383:1813-26.

8. WHO Solidarity Trial Consortium; Pan H, Peto R, Henao-Restrepo AM, Preziosi MP, Sathiyamoorthy V, et al. Repurposed antiviral drugs for Covid-19 - Interim WHO solidarity trial results. N Engl J Med 2021;384:497-511.

9. Statement from the RECOVERY Trial Chief Investigators, on 15 January 2021. Available from: https://www.recoverytrial.net/ news/statement-from-the-recovery-trial-chief-investigators- 15 -january-2021-recovery-trial-closes-recruitment-to-convalescentplasma-treatment-for-patients-hospitalised-with-covid-19. [Last accessed on 2021 Mar 01]. 